DOI: $10.3901 / J M E .2019 .07 .155$

\title{
基于中轴线的曲面网格质量优化
}

\author{
彭威 1,2 吉卫喜 1,2
}

(1. 江南大学机械工程学院 无锡 214122;

2. 江苏省食品先进制造装备技术重点实验室 无锡 214122)

\begin{abstract}
摘要: 网格质量对数值模拟的精度和效率有着重要影响, 为了提升曲面网格质量, 提出一种基于中轴线的曲面网格质量优化 算法, 由几何特征保持和节点光顺操作组成。采用法向张量投票理论对网格节点进行分类, 保证了原始网格的几何特征。节 点光顺操作通过不断的调整节点位置来提升网格质量, 直到网格质量的变化量小于给定的阈值。针对光滑节点, 采用二分法 求解邻域多边形中轴上的最优中心点, 并将该最优中心点投影回三维网格上, 折痕点的最优位置则通过二次多项式插值法求 得。应用实例表明，该方法在保证网格几何特征的基础上，较为明显地提升了曲面网格的质量。
\end{abstract}

关键词: 曲面网格; 网格质量; 网格光顺; 中轴线

中图分类号: TP391

\section{Surface Mesh Quality Improvement Based on Medial Axis}

\author{
PENG Wei ${ }^{1,2}$ JI Weixi ${ }^{1,2}$
}

(1. School of Mechanical Engineering, Jiangnan University, Wuxi 214122;

2. Jiangsu Key laboratory of Advanced Food Manufacturing Equipment and Technology, Wuxi 214122)

\begin{abstract}
Mesh quality plays an important role in the accuracy and efficiency of numerical simulation. In order to improve the quality of surface mesh, a mesh quality improvement algorithm based on medial axis is proposed, which is composed of geometric feature preservation and node smoothing operations. The normal tensor voting theory is used to classify the surface nodes, which can accurately preserve the geometric features of the original mesh. The node smooth operation improves the mesh quality by constantly adjusting the node position until the change of the mesh quality is less than the given threshold. For smoothing node, the optimal point on the medial axis of the surrounding polygon is obtained by dichotomy, and map the optimal point to original surface mesh, while the optimal location of the crease point is obtained by quadratic polynomial interpolation method. The application examples show that the method can improve the quality of the surface mesh on the basis of ensuring the geometric features of the original mesh.
\end{abstract}

Key words: surface mesh; mesh quality; mesh smoothing; medial axis

\section{0 前言}

曲面网格在数字几何处理和数值模拟中有着广 泛的应用。在数字几何处理中, 网格质量是影响模 型光顺性和特征表达的关键因素。在数值模拟中, 数值解的逼近精度和计算效率在很大程度上取决于 网格质量。质量差的单元会显著增加刚度矩阵的条 件数, 从而降低求解结果的准确性、稳定性和求解 效率。因此, 这就需要曲面网格质量优化算法对其 进行优化以达到数值计算和数字几何处理对网格质

* 国家自然科学基金(11402264)和中央高校基本科研业务费专项资金 (JUSRP116026, JUSRP51732B)资助项目。20180507 收到初稿, 20181115 收到修改稿
量的要求。

曲面网格质量优化方法根据网格拓扑是否变化 可以分为拓扑修改法和拓扑保持法两大类。拓扑修 改方法主要是基于网格拓扑改变操作, 即边/面交换 和节点插入/删除操作, 由于四边形网格连接关系的 特殊性和复杂性, 拓扑变换方法实现复杂, 适用性 较差。因此，本文只关注网格拓扑关系保持不变的 网格光顺方法。目前, 曲面网格光顺邻域应用最广 泛的是 Laplacian 平滑技术 ${ }^{[1]}$, 其基本思想是将节点 迭代地移动到其邻域的几何中心。Laplacian 平滑法 具有简单高效的优点, 但对具有凹特征模型进行网 格优化时, 可能会导致网格质量恶化, 甚至产生无 效的单元。为了克服 Laplacian 平滑的缺点, FREITAG $^{[2]}$ 提出了一种智能 Laplacian 平滑算法, 只 
有当相邻网格的质量得到改善时才会发生节点移 动。WANG 等 ${ }^{[3]}$ 提出了基于最大内接圆(Maximum inscribed circle, MIC)的网格光顺算法, 将节点重新 定位分配到其邻域多边形的内接圆圆心位置上, 当 节点邻域多边形为凹多边形时, 该方法得到的网格 质量较差。VARTZIOTIS 等 ${ }^{[4]}$ 采用基于单元线性规 则化变换的几何单元变换法(GETMe)光顺曲面网 格, 该方法可以实现二维网格的优化, 但不能保证 三维曲面网格的质量改善和几何特征保持。上述基 于几何操作的曲面网格优化技术往往忽略了三维曲 面网格的几何特征, 无法保证和原始模型之间的形 状精度。基于优化的网格光顺的基本思想是最小化 或最大化由不同的网格质量度量所构建的能量函 数。 ZHOU 等 ${ }^{[5]}$ 提出了一种基于角度的局部网格优 化方法, 通过求解扭转能量方程使得节点对应的相 邻角度处于最优比例关系, 减少了产生无效单元的 风险。王跃先等 ${ }^{[6]}$ 基于单元畸变能建立节点位移量 的目标函数, 通过最小化该线性能量方程求得节点 位移从而实现局部网格的优化。陈涛等 ${ }^{[7]}$ 以单元最 小角和戔曲的四边形网格质量评价标准为优化目 标, 采用小种群遗传算法进行求解, 从而对网格形 态质量进行优化。文献[8]提出了最优 Delaunay 三角 化的优化方法, 将插值误差作为优化目标, 反复地 将每个节点在局部区域重新定位来减少插值误差, $\mathrm{GAO}$ 等 ${ }^{[9]}$ 将该方法拓展到三维曲面网格, 实现了曲 面网格的优化。LIU 等 ${ }^{[10]}$ 采用整体 Laplacian 算子对 曲面网格进行优化, 通过求解稀疏线性方程组来求 解该能量最小化问题。基于优化的方法能产生较好 的结果, 但由于要求解复杂的能量方程, 计算量很 大。因此, 在保证模型形状精度的前提下, 如何快 速有效的提高曲面网格的质量是目前必须解决的问 题。本文提出了一种基于中轴线的曲面网格质量优 化算法, 首先采用法向张量投票法将曲面节点进行 分类, 针对不同类型的节点进行位置优化, 直到网 格质量的变化满足给定的收玫条件。

\section{1 算法描述}

网格质量是数值模拟的关键, 网格质量优化的 目标是在精确地表示原始模型的基础上, 尽可能地 输出更为规则的网格, 以满足有限元计算和后续实 体网格生成的要求。本文提出的网格质量优化方法 是一种基于迭代几何操作的方法, 将节点不断的重 定位到其邻域空间内的最优位置来提升曲面网格的 质量。为了保持原始模型的特征和形状精度, 采用 法向张量投票法将所有曲面节点分为以下三种类
型: (1)光滑节点; (2)折痕节点; (3)角节点。根据不 同的节点类型分别进行局部网格的优化, 角节点被 限制在原始位置不可移动, 因此主要针对光滑节点 和折痕节点。折痕节点被限制在与其相连接的两条 折痕边上移动, 因此将折边点重定位到折边的中点 上以提高其局部网格质量。当节点为光滑节点时, 由于节点的曲率较低, 节点的重定位问题可以转化 为寻找二维平面多边形最优中心的问题。平面多边 形最优中心处于多边形的中轴线上, 但最大内接圆 圆心并不一定能得到最优的网格质量。为此, 本文 采用二分法求解位于多边形中轴线上最优中心点, 提升局部网格的质量。上述节点位置优化过程是一 个迭代过程, 当优化前后的网格质量变化小于给定 的误差量或迭代次数达到最大迭代步时则终止迭 代。本文提出的曲面网格质量优化算法流程如下。

(1) 节点分类。

对每个网格节点 $v$ 执行下列步骤。

1）计算节点相邻单元的法向 $\boldsymbol{n}$, 构建局部法向 张量矩阵 $\boldsymbol{T}$ 。

2) 根据张量矩阵 $\boldsymbol{T}$ 的特征值关系将节点 $v$ 进行 归类(角节点、折痕节点和光滑节点)。

(2) 网格质量优化。

对每个网格节点 $v$ 执行下列步骤。

1) 如果节点类型为折痕节点, 将节点 $v$ 定位到 其连接折线插值曲线的中心位置。

2) 如果节点类型为光滑节点, 将其邻域多边形 投影到二维平面, 采用二分法将节点 $v$ 重定位到其 平面邻域多边形的中轴线上, 最后投影回到三维曲 面上。

(3) 收敛判断。

1) 计算第 $t$ 迭代步的网格质量 $J\left(M_{t}\right)$ 。

2) 给定收玫因子 $\sigma$, 如果 $\left|J\left(M_{t}\right)-J\left(M_{t-1}\right)\right|<\sigma$ 或 达到给定的最大迭代步则迭代终止, 否则返回步 骤(2)。

\section{2 曲面网格质量优化}

\section{1 节点分类}

尖锐的几何特征是精确表征原始几何的关键, 为了在网格光滑过程中保持原始模型的特征, 将所 有网格节点划分为光滑节点、折痕节点和角节点三 种类型。光滑节点在邻域内曲率较低, 被限制在其 周围的曲面网格上, 而折痕节点只允许沿着折痕的 方向移动, 而角节点被限制在初始位置。本文利用 法向张量投票理论 ${ }^{[11]}$ 对节点进行识别。对于曲面网 格 $M$ 上的节点 $v_{i}$, 其一阶邻域网格可以表示为 $N_{f}\left(v_{i}\right)$, 
$\left|N_{f}\left(v_{i}\right)\right|$ 是相邻网格的数量。顶点 $v_{i}$ 的法向投票张量 $\boldsymbol{T}$ 定义如下

$$
\boldsymbol{T}_{v i}=\sum_{l=1}^{\left|N_{s_{f}}\left(v_{v}\right)\right|} \omega_{l} \boldsymbol{n}_{l} \boldsymbol{n}_{l}^{\mathrm{T}}
$$

式中 $n_{l}$ 一网格的单位法矢量;

$\omega_{l}$ —单位法矢量对应的权重。

$$
\omega_{l}=\frac{A\left(f_{i, l}\right)}{A_{\max }} \exp \left(-\frac{g_{l}}{(\sigma / 3)}\right)
$$

式中 $A\left(f_{i, l}\right)$ 一单元 $f_{i, l}$ 的面积;

$A_{\max }-v_{i}$ 一阶邻域网格中最大的面积;

$g_{l}-v_{i}$ 到单元 $f_{i, l}$ 中心的距离;

$\sigma-v_{i}$ 一阶邻域空间包围正方体的边长。

张量矩阵 $\boldsymbol{T}$ 是一个半正定对称矩阵, 可以采用 特征值分析法对其进行分解, 并根据特征值之间的 关系来确定节点 $v_{i}$ 的类型。假设 $\lambda_{1} \geqslant \lambda_{2} \geqslant \lambda_{3} \geqslant 0$ 是它 的三个特征值, $\boldsymbol{d}_{1}, \boldsymbol{d}_{2}$ 和 $\boldsymbol{d}_{3}$ 是特征值所分别对应的 特征矢量。令 $S S=\lambda_{1}-\lambda_{2}, S e=\lambda_{2}-\lambda_{3}, S c=\lambda_{3}$, 节点 $v_{i}$ 的分类规则如下所示

$$
\max (S s, \varepsilon S e, \varepsilon \eta S c)=\left\{\begin{array}{l}
S s\left(v_{i} \text { 是光滑点 }\right) \\
\varepsilon S e\left(v_{i} \text { 是 } \boldsymbol{d}_{3} \text { 方向上的折痕点 }\right) \\
\varepsilon \eta S c\left(v_{i} \text { 是角点 }\right)
\end{array}\right.
$$

式中, $\varepsilon$ 和 $\eta$ 是用来平衡噪声点和判断折边点的敏 感参数, 本文都设为 4.0 .

\section{2 网格质量优化}

网格质量优化的目标是将每个节点重定位到新 的位置来提升局部网格的质量, 本文针对不同类型 的节点采用不同的优化策略。对于光滑节点, 由于 节点处的曲率较小, 可以将节点邻域多边形直接投 影到节点法平面上, 节点位置优化因此转化为在平 面多边形内寻找最优节点的问题。节点 $v_{i}$ 的法矢量 $\boldsymbol{n}_{i}$ 由与节点 $v_{i}$ 相邻的单元法矢量加权得到

$$
\boldsymbol{n}_{i}=\frac{\sum_{f_{i, l} \in N_{f}\left(v_{i}\right)} A\left(f_{i, l}\right) n_{l}}{\sum_{f_{i, l} \in N_{f}\left(v_{i}\right)} A\left(f_{i, l}\right)}
$$

式中 $\boldsymbol{n}_{l}$ 一相邻网格 $f_{i, l}$ 的单位法矢量;

$A\left(f_{i, l}\right)$ 一单元 $f_{i, l}$ 的面积。

网格最小角度对网格质量起了决定性作用, 理 想的平面多边形中心点可以最大化多边形内网格的 最小角。因此, 多边形内到边界最小距离最大的点 被认为是多边形的最优中心点, 而多边形中轴线 ${ }^{[12]}$ 是多边形内到边界距离相等的点的集合, 本文认为 该中心点处于多边形的中轴线上。为此, 本文提出 了基于多边形中轴线的最优中心点求解方法, 采用 二分法求解多边形中轴线上的最优中心点。给定节 点 $v_{i}$ 及其平面邻域多边形 $P$, 最优中心点求解步骤
如下。

(1) 判断多边形 $P$ 的凹凸属性。由于多边形的 凹凸属性对最优中心点的寻找非常关键, 首先需要 判断多边形的凹凸属性。

(2) 转化多边形 $P$ 为凸多边形。当多边形 $P$ 为 凹多边形, 最优中心点的求解容易出现偏差, 因此 需要将其转化为凸多边形。对于多边形 $P$ 上的凹节 点, 延长其相邻的边, 求延长边与多边形的交点, 根据交点组成新的节点系列, 得到新的多边形, 直 至消除多边形内的所有凹节点(图 3b)。

(3) 求解多边形中轴线的单调区间。求解凸多 边形的中轴线, 该中轴线由一系列直线段构成。为 了获取中轴线上的最优中心点, 需要将中轴线细分 为若干单调区间, 在单个区间内, 点与多边形节点 构成的夹角呈单调增大或减小。对于中轴线上的每 条线段 $\left\{S_{1}, S_{2}\right\}$, 考虑节点与多边形边的夹角, 将其 与多边形 $P$ 上的角平分线求交。如图 1 所示, 对于 节点与多边形节点构成的夹角, 根据圆周角定理, $\angle v_{3} S_{1} v_{2}=\angle v_{3} S_{\mathrm{e}} v_{2}$, 点 $S_{\mathrm{m}}$ 与 $v_{2} v_{3}$ 的夹角 $\angle v_{3} S_{\mathrm{m}} v_{2}$ 大 于 $\angle v_{3} S_{1} v_{2}$, 因此可以认为直线段上的点和圆心 $\boldsymbol{c}$ 的距离越小, 角度越大。由于圆心 $\boldsymbol{c}$ 的位置在边界 中垂线上变化, 为了简化计算, 本文将圆心 $c$ 固定 在边界的中点位置, 因此将多边形 $P$ 的边界中点 投影到线段 $\left\{S_{1}, S_{2}\right\}$ 上求得角度变化的近似拐点。 若存在交点和投影点, 则按顺序将线段 $\left\{S_{1}, S_{2}\right\}$ 细 分(图 3b)。

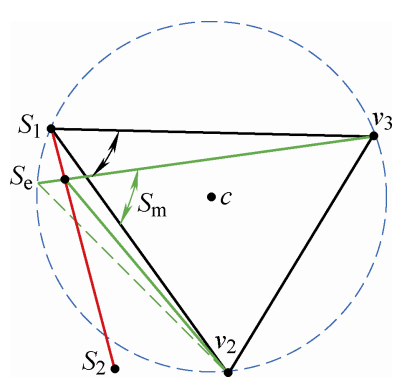

图 1 圆周角示意图

（4）采用二分法求解最优中心点。对于中轴线 上的细分线段 $\left\{\alpha_{\mathrm{S}}, \alpha_{\mathrm{E}}\right\}$, 由于细分线段上的点与多边 形构成的夹角在线段上呈现单调递增或递减的 变化, 采用二分法求解最优中心点的步骤如下(如 图 2)。

(1) 令 $s m_{1}=\left(\alpha_{\mathrm{S}}+\alpha_{\mathrm{E}}\right) / 2$, 求得 $s m_{1}$ 处点与多边形 构成的最小夹角。

(2) 根据最小角度的单调变化趋势更新线段区 间。如果角度单调递增, 令 $\alpha_{\mathrm{S}}=s m_{1}$, 如果角度单 调递减 $\alpha_{\mathrm{E}}=s m_{1}$, 返回步骤(1)。

终止条件: 线段区间小于给定误差或前后迭代步 
的最小角度属于同一夹角且变化值小于给定误差。

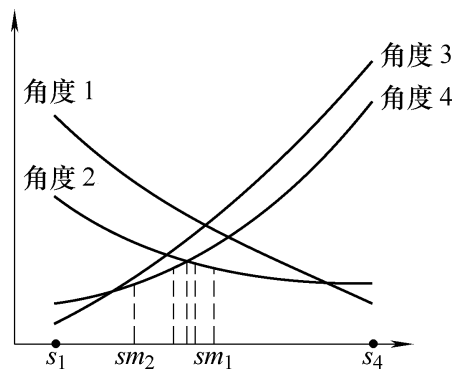

图 2 二分法求解最优多边形中心

图 3 所示为凹多边形网格光顺实例及其与最大 内接圆 (MIC) $)^{[3]}$ 和 Laplacian 光顺 ${ }^{[1]}$ 的比较。图 3a 为 节点 $v_{i}$ 的平面邻域凹多边形 $P=\left\{v_{1}, v_{2}, \ldots, v_{7}\right\}$, 经过 转化后的凸多边形为 $P=\left\{v_{2}, v_{8}, v_{5}, v_{6}, v_{9}\right\}$ 及其中轴线
如图 $3 \mathrm{~b}$ 所示, 中轴线节点为 $\left\{S_{1}, S_{2}, S_{3}\right\}$ 。 $\mathrm{S}_{4}$ 是 $v_{3}$ 处 角平分线和直线段 $\left\{S_{1}, S_{2}\right\}$ 的交点，而边界 $\left\{v_{2}, v_{3}\right\}$ 的 中点 $m_{2}$ 在 $\left\{S_{1}, S_{2}\right\}$ 的投影点为 $\mathrm{S}_{5}$ 。因此 $\left\{S_{1}, S_{2}\right\}$ 在被 细分为若干单调的细分区间, 最终求得的最优中心 点在图 $3 \mathrm{c}$ 中的 $v_{\mathrm{S}}$ 处, 而最大内接圆和 Laplacian 光 顺得到的结果如图 $3 \mathrm{~d}$ 和 $3 \mathrm{e}$ 所示。图 3a、3c、3d 和 $\mathrm{e}$ 中对应的网格最小角度分别为 $11.4^{\circ} 、 20.9^{\circ} 、 1.16^{\circ}$ 和 $5.66^{\circ}$ 。图 4 为凸多边形网格光顺实例。图 $4 \mathrm{a}$ 是 以节点 $v_{i}$ 为中心的平面网格, 其最小角度是 $10.3^{\circ}$, 它的中轴线如图 $4 \mathrm{~b}$ 所示。本文方法、最大内接圆和 Laplacian 方法得到的光顺结果分别如图 4c、4d 及 $4 \mathrm{e}$ 所示, 其中最小角度分别为 $30.5^{\circ} 、 20.1^{\circ}$ 和 $25.7^{\circ}$ 。 因此, 从图 3 和 4 可知, 在凹多边形和凸多边形的 情况下，本文方法都可以得到更好的光顺效果。

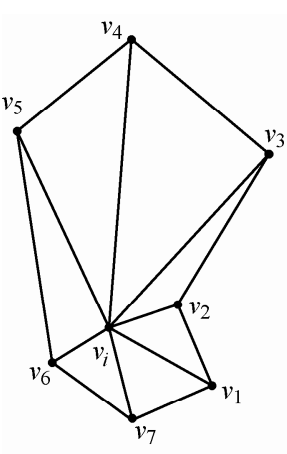

(a) 原始多边形

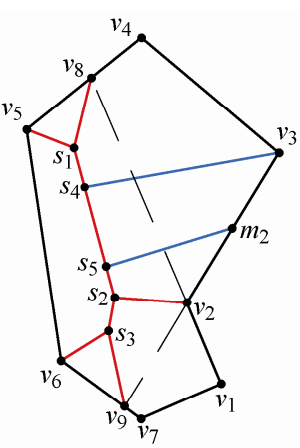

(b) 多边形中轴线

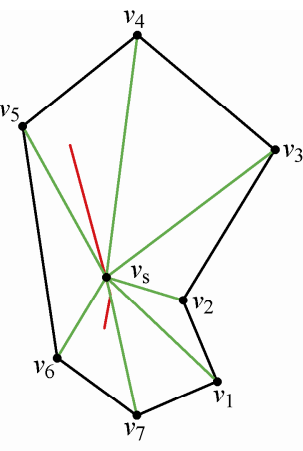

(c) 本文方法

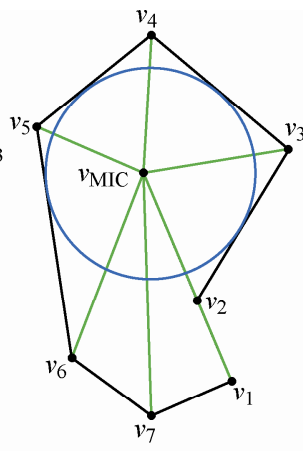

(d) 最大内接圆

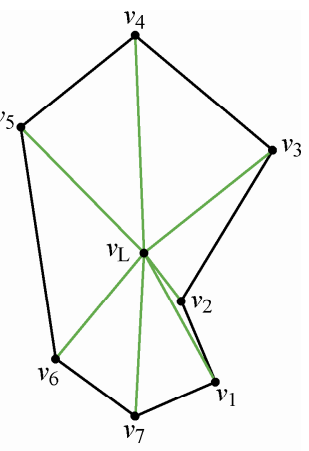

(e) Laplacian

图 3 凹多边形网格光顺

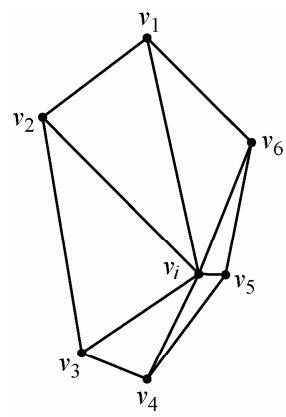

(a) 原始多边形

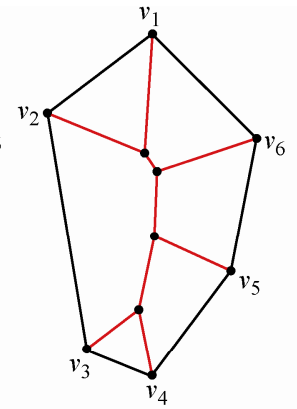

(b) 多边形中轴线

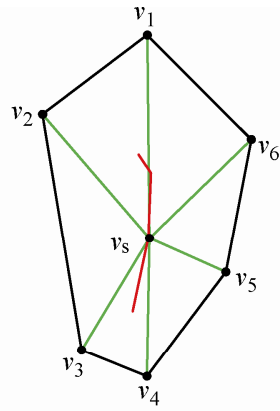

(c) 本文方法

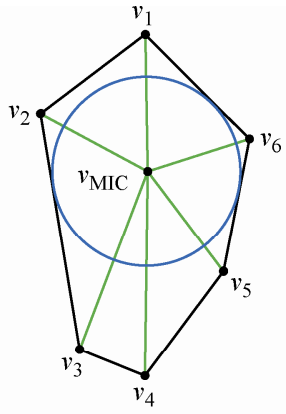

(d) 最大内接圆

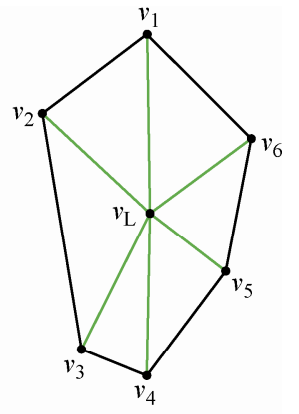

(e) Laplacian

图 4 凸多边形网格光顺

对于折痕节点 $v_{i}$, 只允许其在相邻折痕线内移 动, 首先根据网格边和矢量 $\boldsymbol{d}_{i}$ 的最大最小夹角识别 两条相连折痕线, 分别表示为: $e_{i, c 1}=\min \left\langle\boldsymbol{e}, \boldsymbol{d}_{i}>\right.$ 和 $e_{i, c 2}=\max <\boldsymbol{e}, \boldsymbol{d}_{i}>\left(0 \leqslant<\boldsymbol{e}, \boldsymbol{d}_{i}>\leqslant \pi\right)$ 。由于折痕线 代表了模型的几何特征, 为了精确地表达原始模型 同时提升折痕点处的网格质量, 这里采用二次多项 式插值法生成折痕曲线, 并选择该曲线的中点作为 $v_{i}$ 的优化位置(图 5)。

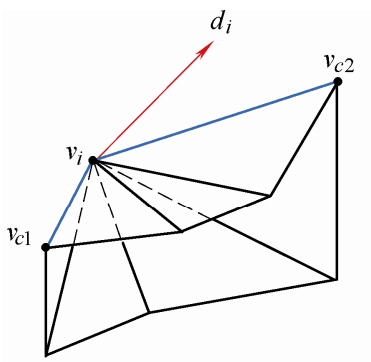

(a) 折痕点

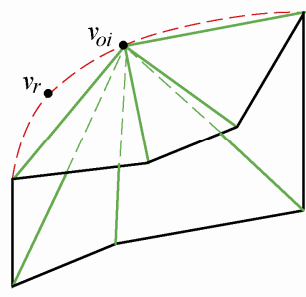

(b) 折痕点光顺

图 5 折痕点网格光顺 


\section{3 优化实例及分析}

采用 vs2008 以及 Open CASCADE 开源平台开 发了相应的网格优化程序。为了验证本文算法的正 确性, 选用缩放雅可比(Scaled Jacobian)作为曲面网 格质量评价的标准 ${ }^{[13]}$, 该标准适用于由三角形和四 边形单元组成的曲面网格。缩放雅可比是有限元网 格质量的基本评判标准之一, 对于单元 $f=\left(v_{1}, \ldots\right.$, $\left.v_{4}\right)$, 节点 $v_{i}$ 的缩放雅可比值为 $J\left(f_{v_{i}}\right)=\left|\boldsymbol{v}_{i+1} \boldsymbol{v}_{i} \times \boldsymbol{v}_{i-1} \boldsymbol{v}_{i}\right|$, 单元 $f$ 中所有节点的缩放雅可比最小值即为单元 $f$ 的缩放雅可比值, 有限元计算容许的缩放雅可比值 一般在 $[0.25,1]$ 范围内。本文中, 定义缩放雅可比最 小值 $J_{\text {min }}=\min _{j \in\{1, \cdots, n\}} J\left(f_{j}\right)$, 平均值 $J_{\text {mean }}=1 / n \sum_{j=1}^{n} J\left(f_{j}\right)$, 其中 $n$ 为曲面网格数量。同时为了验证本文算法的
高效性, 本文与最大内接圆(MIC) ${ }^{[3]}$ 和 Laplacian 方 法 $^{[1]}$ 进行了比较, 这两种方法都包含了本文的节点 分类和折痕点网格光顺操作, 以此来保证原始网格 的特征。

实例一为摇臂零件的网格质量优化实例, 图 $6 \mathrm{a}$ 所示为根据缩放雅可比值着色的原始三角网格。显 然, 原始网格含有很多低质量网格, 其最小缩放雅 可比最小值 $J_{\min }=0$ 。采用 Laplacian、最大内接圆和 本文网格优化算法后得到的曲面网格如图 $6 \mathrm{~b} 、 6 \mathrm{c}$ 及 $6 \mathrm{~d}$ 所示。原始网格和通过本文算法优化后的网格 之间的距离偏差分布如图 6e 所示, 其中最大的距离 偏差是 0.24 。为了比较采用不同方法优化后的摇臂 模型网格质量，图 7 展示了不同优化结果的网格质 量分布直方图。从图中可知, 本文方法可以有效地 提高曲面网格的网格质量, 并能获得比 Laplacian 和最大内接圆更好的优化结果。

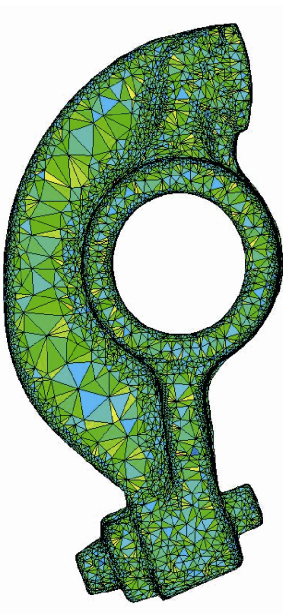

(a) 原始网格

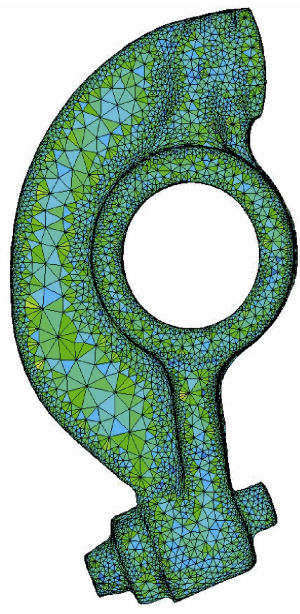

0

(b) Laplacian

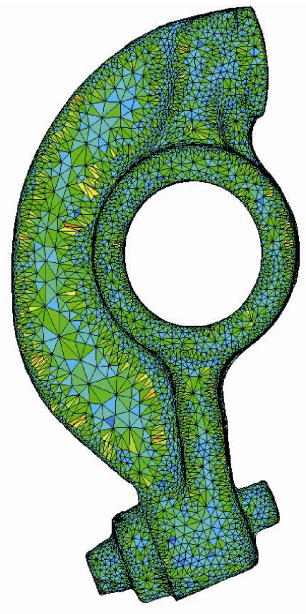

0.5

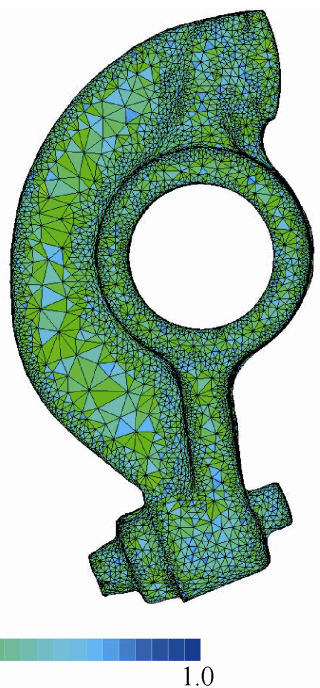

(d) 本文方法

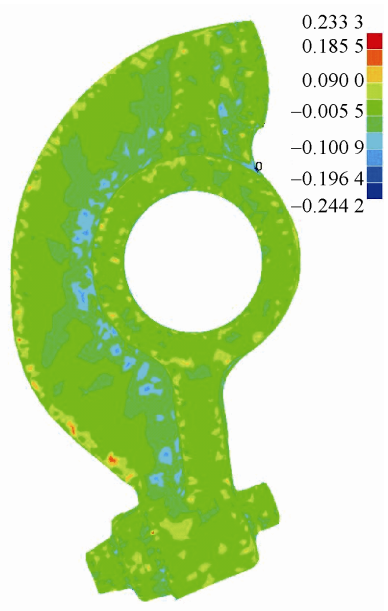

(e) 误差云图

图 6 摇臂曲面网格优化

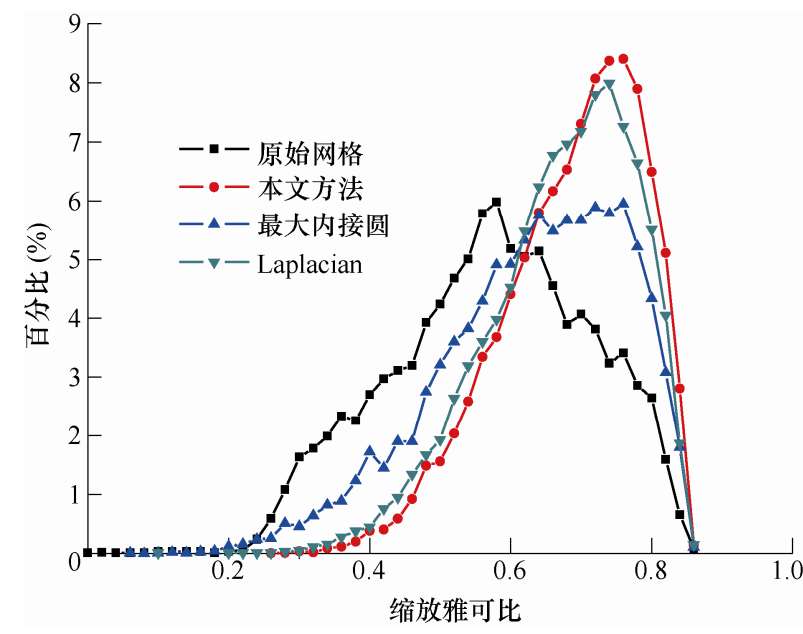

图 7 摇臂曲面网格质量优化对比

图 8a 所示的 Fandisk 模型是从点云重建而得到 的三角网格模型, 其中包含很多尖锐的三角形, 最 小缩放雅克比值接近 0 。采用本文提出的网格优化 算法后得到的曲面网格如图 $8 \mathrm{~b}$ 所示, 原始网格和优 化后网格的距离偏差如图 8c 所示, 图中在尖锐边界 处的较大误差可能是节点类型判断时由于噪声点所 引起的。图 9 绘制了初始网格和通过各种优化方法 优化后得到的网格的质量分布直方图。通过本文方 法, 最小网格质量提高到了 0.22 , 整体网格质量提 高到 0.71 。 


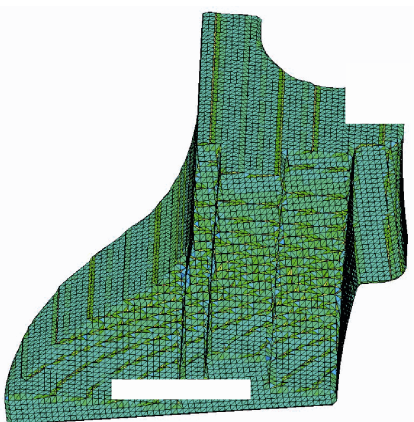

(a) 原始网格

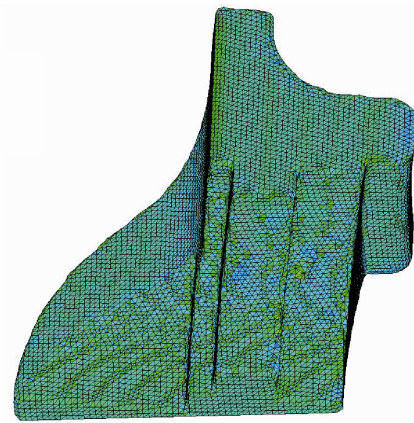

(b) Laplacian

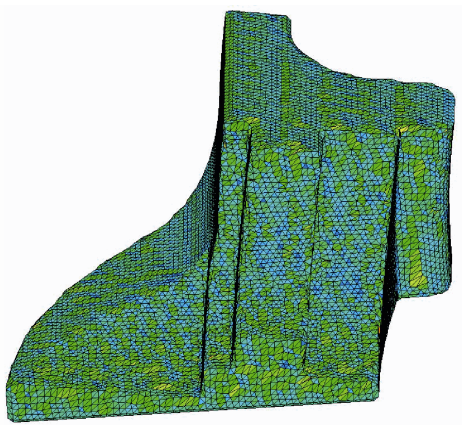

(c) 最大内接圆

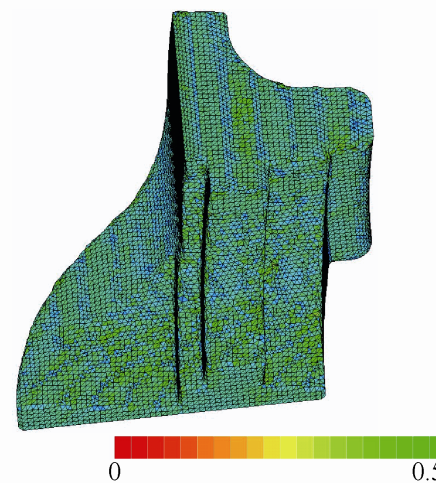

(d) 本文方法

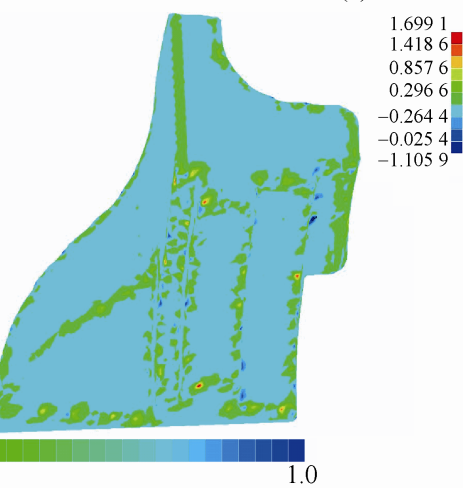

(e) 误差云图

图 8 fandisk 曲面网格质量优化

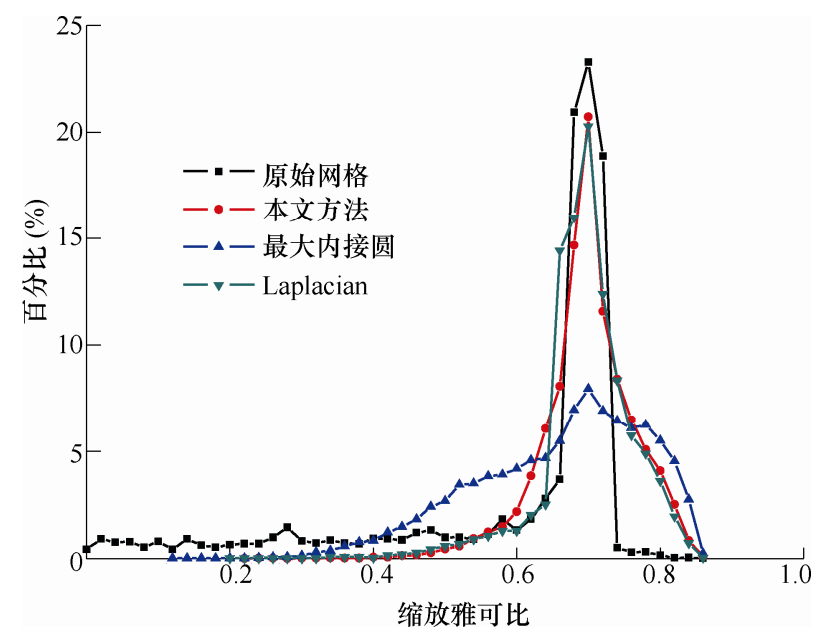

图 9 Fandisk 网格质量优化对比

实例三是航空发动机叶片曲面网格优化实例。 图 10a 为叶片的原始网格, 包含 28570 个四边形单 元和 1070 个三角形单元, 图 10b 所示为本文网格 质量优化算法优化后的曲面网格, 图 $10 \mathrm{c}$ 为原始模 型和优化后模型的距离偏差分布图。图 11 比较了叶 片的曲面网格采用不同优化方法优化前后的网格质 量。由图 10a 中可看出原始曲面网格在一些尖锐位 置网格质量较差, 最小缩放雅可比 $J_{\min }=0.05$, 低于 通用有限元计算程序要求的最低值。通过本文方法 优化后, 尖锐位置网格质量得到改善, 缩放雅可比 最小值提高到 $J_{\mathrm{min}}=0.33$, 使得网格处于有限元数值 计算可接受范围。因此, 优化后的叶片曲面网格可 以进行有限元数值计算。
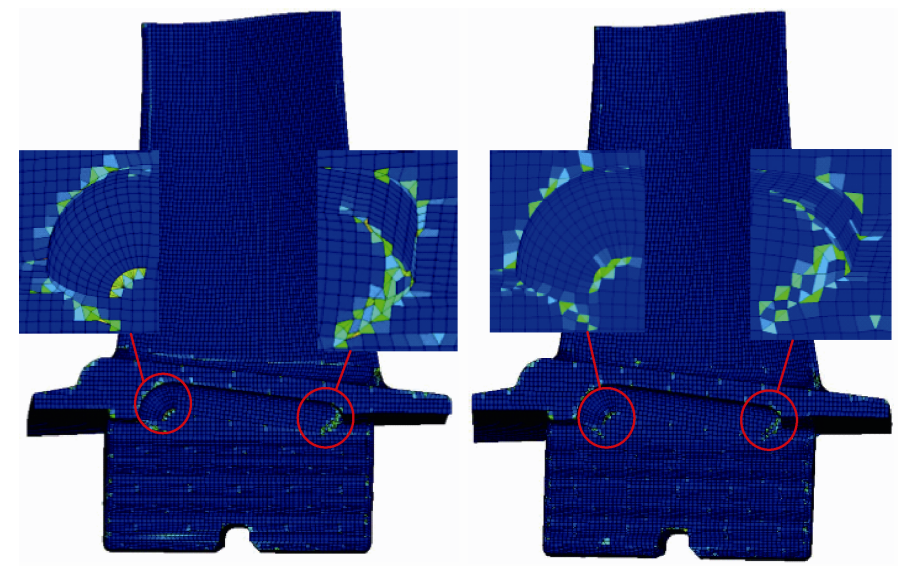

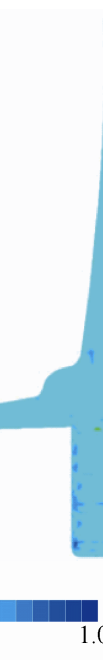

(b) 本文优化结果

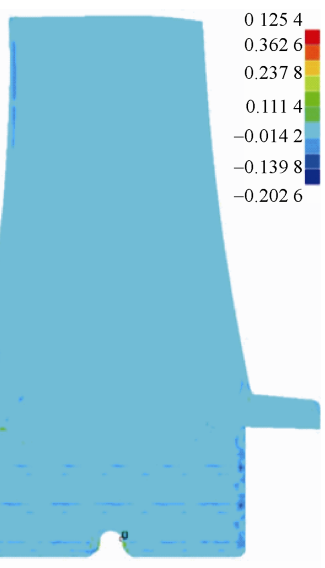

1.0

(a) 原始网格

(c) 误差云图

图 10 叶片曲面网格质量优化 


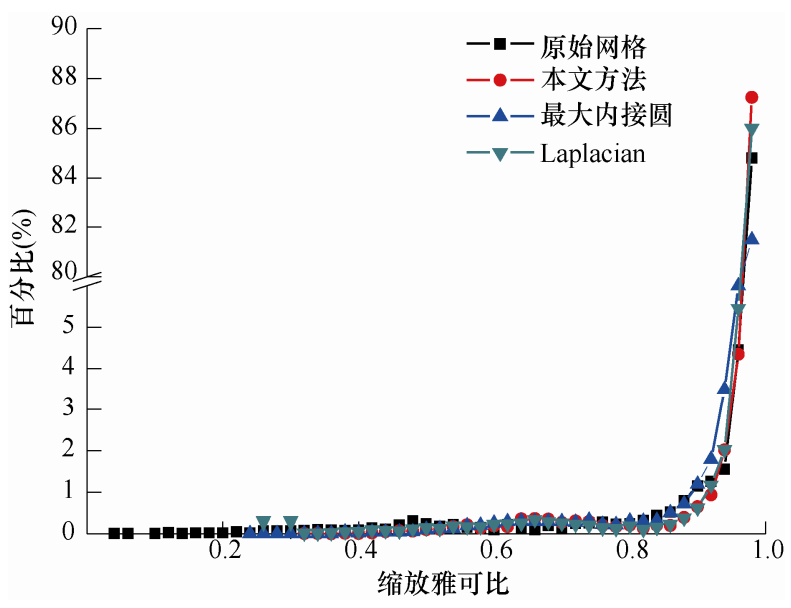

图 11 叶片曲面网格质量优化对比

表 1 所示为曲面网格优化结果统计, 表 2 为采 用不同优化方法所需的计算时间。从优化结果和计 算时间对比情况可知, 本文方法由于需要计算凸多 边形的中轴线, 耗时多于 Laplacian 方法, 与最大内 接圆方法耗时大致相同, 但能获得优于上述两种方 法的结果。

表 1 优化结果统计

\begin{tabular}{|c|c|c|c|c|c|c|}
\hline & \multicolumn{2}{|c|}{ 摇臂 } & \multicolumn{2}{|c|}{ Fandisk } & \multicolumn{2}{|c|}{ 叶片 } \\
\hline & $J_{\min }$ & $J_{\text {mean }}$ & $J_{\min }$ & $J_{\text {mean }}$ & $J_{\min }$ & $J_{\text {mean }}$ \\
\hline 原始模型 & 0 & 0.58 & 0.02 & 0.60 & 0.05 & 0.97 \\
\hline 本文算法 & 0.26 & 0.69 & 0.22 & 0.71 & 0.33 & 0.98 \\
\hline Laplacian & 0.11 & 0.68 & 0.20 & 0.70 & 0.25 & 0.98 \\
\hline 最大内接圆 & 0.08 & 0.64 & 0.12 & 0.67 & 0.26 & 0.98 \\
\hline
\end{tabular}

表 2 计算时间对比

\begin{tabular}{lccc}
\hline \multirow{2}{*}{ 模型 } & \multicolumn{3}{c}{ 计算时间 $/ \mathrm{s}$} \\
\cline { 2 - 4 } & 本文算法 & Laplacian & 最大内接圆 \\
\hline 摇臂 & 1.97 & 1.28 & 1.87 \\
Fandisk & 3.21 & 1.14 & 3.16 \\
叶片 & 2.01 & 1.34 & 1.86 \\
\hline
\end{tabular}

\section{4 结论}

(1) 采用法向张量投票理论对网格节点进行 分类, 保证了网格质量优化过程中模型的几何特征 不变。

(2) 采用二分法求解平面多边形中轴线上的最 优中心点, 提升了局部网格质量, 通过与最大内接 圆(MIC)和 Laplacian 光顺的比较, 证明该方法可以 较为明显地提高曲面网格质量。

应用实例表明, 本文方法在保证原始模型几何 特征的前提下, 改善了曲面网格的质量, 所得结果 优于 Laplacian 和最大内接圆(MIC), 优化后的网格 质量满足了有限元计算的要求。

\section{参 考 文 献}

[1] FIELD D A. Laplacian smoothing and Delaunay triangulations[J]. Communications in Applied Numerical Methods, 1988, 4(6): 709-712.

[2] FREITAG L A. On combining Laplacian and optimization based mesh smoothing techniques[C]. //Trends in Unstructured Mesh Generation, 1997, Evanston, Illinois. ASME 1997: 37-43.

[3] WANG J, YU Z. Quality mesh smoothing via local surface fitting and optimum projection[J]. Graphical Models, 2011, 73(4): 127-139.

[4] VARTZIOTIS D, WIPPER J, PAPADRAKAKIS M. Improving mesh quality and finite element solution accuracy by GETMe smoothing in solving the Poisson equation[J]. Finite Elements in Analysis \& Design, 2013, 66(66): 36-52.

[5] ZHOU T, SHIMADA K. An angle-based approach to two-dimensional mesh smoothing[C] //In: Proceedings of the 9th International Meshing Roundtable, 2000, New Orleans. 2000: 373-384.

[6] 王跃先，陈军，阮雪榆. 金属塑性成形有限元仿真中的 网格质量优化 $[\mathrm{J}]$. 塑性工程学报, 2002, 9(1): 10-13. WANG Yuexian, CHEN Jun, RUAN Xueyu. Mesh optimization in FEM stimulation to metal plastic forming[J]. Journal of Plasticity Engineering, 2002, 9(1): 10-13.

[7] 陈涛, 李光耀. STL 格式文件的四边形网格剖分与网格 光顺 [J]. 中国机械工程，2009，20(5)：522-528.

CHEN Tao, LI Guangyao. Quadrilateral mesh generation based on STL file format model and mesh smoothing $[\mathrm{J}]$. China Mechanical Engineering, 2009， 20(5): 522-528.

[8] CHEN L, HOLST M. Efficient mesh optimization schemes based on optimal Delaunay triangulations $[\mathrm{J}]$. Computer Methods in Applied Mechanics and Engineering, 2011, 200(9): 967-984.

[9] GAO Z, YU Z, HOLST M. Feature-preserving surface mesh smoothing via suboptimal Delaunay triangulation[J]. Graphical Models, 2013， 75(1): 23-38.

[10] LIU L, TAI C L, JI Z, et al. Non-iterative approach for global mesh optimization[J]. Computer Aided Design, 2007, 39(9): 772-782.

[11] KIM H, HAN K, LEE K. Feature detection of triangular meshes based on tensor voting theory[J]. Computer Aided Design, 2009, 41(1): 47-58.

[12] BERG M, KREVELD M, OVERMARS M, et al. 计算 几何: 算法与应用 $[\mathrm{M}]$. 邓俊辉译. 北京: 清华大学出 版社, 2005.

BERG $\mathrm{M}$, KREVELD $\mathrm{M}$, OVERMARS $\mathrm{M}$, et al. 
Computational geometry: Algorithms and applications[M]. Tramslated by DENG Junhui. Beijing: Tsinghua University Press, 2015.

[13] KNUPP P M. Achieving finite element mesh quality via optimization of the Jacobian matrix norm and associated quantities. Part I-a framework for surface mesh optimization[J]. International Journal for Numerical
Methods in Engineering, 2000， 48(3): 401-420.

作者简介: 彭威(通信作者), 男, 1984 年出生, 博士, 讲师。主要研究 方向为有限元计算, 数字化制造。

E-mail: weipeng@jiangnan.edu.cn

吉卫喜, 男, 1961 年出生, 博士, 教授, 博士研究生导师。主要研究方 向为数字化制造, 智能制造。

E-mail: jiweixi@jiangnan.edu.cn

\section{第 8 届上银优秀机械博士论文奖——佳作奖}

\section{复杂型面薄壁回转体零件旋印电解加工基础研究}

作 者: 王登勇

毕业学校: 南京航空航天大学

指导教师: 朱荻, 朱增伟

机匣是一种大型薄壁回转体零件, 是航空发动机的核心部件, 起着连接、承力、包容等作用。由于机匣壁薄、外形结构 复杂、材料去除量大等特点, 采用切削加工等制造手段普遍存在加工精度差、效率低等瓶颈问题。为解决机匣零件的制造难 题, 本文提出了旋印电解加工技术, 采用带有窗口的回转体工具电极, 通过工件与工具的同步对转, 实现机匣复杂凹凸型面 的一次性无变形加工, 该技术对于薄壁机匣零件加工具有独特的优势。本文针对旋印电解加工中的若干基础问题开展研究, 主要研究内容包括以下几点。

(1) 开展阳极工件型面动态成形规律研究。建立旋印电解加工数学模型, 得出材料去除速率、加工间隙的变化规律, 开 展凸台轮廓成形仿真, 掌握了凸台轮廓成形规律。

(2) 揭示对转脉动态下材料电化学溶解机理。根据旋印电解加工脉动态电流分布特性, 开展溶解试验研究, 建立 GH4169 材料在大间歇状态下的溶解特性模型, 揭示了材料蚀除速率与钝化膜破裂时间、电极转速间的变化规律。

（3）提出两种抑制凸台杂散腐蚀方法, 显著提高凸台成形精度。一种是利用在低电流密度处于钝化状态的钝性金属涂层, 来保护凸台表面不受杂散腐蚀; 另一种是通过柔性正电位辅助电极来改变凸台表面的电场分布, 消除凸台表面的杂散电流。

(4) 提出基于阴极窗口运动轨迹优化的回转体阴极工具设计方法。在阳极成形规律研究基础上, 制定了阴极半径、雉角、 窗口尺寸的设计原则，并开发出阴极设计软件。

(5) 研制出旋印电解加工机床装备, 实现薄壁机匣样件加工。对机床运动单元、密封装置、引电装置等关键部件进行设 计, 解决了电极同步旋转、运动部件密封、旋转引电等关键技术。针对某型号航空发动机机匣缩比模型, 制定了工具设计、 工装夹具设计等一整套加工方案, 成功实现薄壁机匣样件的一次性加工成形, 表面粗䊁度可达 $R_{\mathrm{a}} 0.2 \mu \mathrm{m}$, 最小壁厚 $0.96 \mathrm{~mm}$, 壁厚均匀性小于 $0.1 \mathrm{~mm}$, 凸台轮廓精度满足设计要求。 